\title{
Fabrication and Characterisation of Microscale Air Bridges in Conductive Gallium Nitride
}

\author{
Chang Xiong', David Massoubre ${ }^{2}$, Erdan $\mathrm{Gu}^{2}$, Martin D. Dawson ${ }^{2}$ and Ian M. \\ Watson $^{2} *$ \\ ${ }^{1}$ School of Physics and State Key Laboratory for Artificial Microstructures and \\ Mesoscopic Physics, Peking University, Beijing 100871, People's Republic of China \\ ${ }^{2}$ Institute of Photonics, SUPA, Wolfson Centre, 106 Rottenrow, Glasgow G4 0NW, \\ United Kingdom
}

*Corresponding author: e-mail i.m.watson@strath.ac.uk, fax +44 1415521575

\begin{abstract}
Fabrication and electrical characterisation of microscale air bridges consisting of GaN heavily doped with silicon is described. These were made from GaN-AlInN-GaN epitaxial trilayers on sapphire substrates, in which the AlInN was close to the composition lattice matched to $\mathrm{GaN}$ at $\sim 17 \% \mathrm{InN}$ fraction. The start of the fabrication sequence used inductively coupled plasma etching with chlorine chemistry to define mesas. In situ monitoring by laser reflectometry indicated an AlInN vertical etch rate of $400 \mathrm{~nm} /$ minute, $\sim 70 \%$ of the etch rate of GaN. Processing was completed by lateral wet etching of the AlInN in hot nitric acid to leave GaN microbridges supported between anchor posts at both ends. Deposition of Ti-Au contact pads onto the anchor posts allowed study of the electrical characteristics. At low applied voltages, vertical conduction through the undoped AlInN layers was minimal in comparison with the current path through the Si:GaN bridges. Typical structures showed highly linear current-voltage characteristics at low applied voltages, and had resistances of $1050 \Omega$. The observed resistance values are compared with the predicted value based on materials parameters and an idealised geometry. The microbridges showed damage from Joule heating only at current densities above $2 \times 10^{5} \mathrm{~A} \mathrm{~cm}^{-2}$.
\end{abstract}

\section{PACS numbers:}

07.10.Cm (micromechanical devices)

72.80.Ey (electrical conductivity of III-V semiconductors)

81.05.Ea (fabrication of III-V semiconductors) 


\section{Introduction}

Semiconductors from the GaN family have considerable potential in novel microelectromechanical systems (MEMS). The detailed motivations and MEMS fabrication techniques developed to date for $\mathrm{SiC}$ and the III-nitrides were recently reviewed by Cimalla et al [1]. One key aspect is the suitability of these materials to fabricate devices for service under harsh conditions, including high temperatures and chemically aggressive environments. The piezo- and pyroelectric properties of the wurtzite-phase nitride materials also offer novel possibilities for transduction mechanisms.

The established approaches to fabricating MEMS in silicon and most compound semiconductors rely on sacrificial layer technologies, in which a sacrificial material is removed from under layers retained in the finished device by lateral etching. The lateral etch step can use either wet etching or isotropic dry etching, and usually follows an anisotropic vertical dry etch defining the plan-view shape of the microstructure. Such process sequences enable the fabrication of many different functional microstructures, including beams and cantilevers for use as mechanical resonators, large-area suspended membranes, and air-spaced Bragg mirrors. Hjort reviewed the early development of such 3-dimensional microstructures in non-nitride III-V semiconductors, and stressed the high selectivites $(>100)$ attainable in optimised wet etch processes with many material combinations [2]. Although III-nitride materials offer an exceptionally wide variation of chemical and physical properties within the $(\mathrm{Ga}, \mathrm{In}, \mathrm{Al}) \mathrm{N}$ quaternary system, development of analogous sacrifical layer technologies has been relatively slow. A popular and effective route to undercut microstructures in GaN has actually used bulk silicon micromachining, employing a silicon substrate as the sacrifical material [3-5]. These approaches rely on the complex growth sequences necessary for production of GaN-based epistructures on a highly mismatched substrate, and the retention of exposed silicon in a finished device also negates some advantages of the III-nitrides for service in harsh environments. Sacrifical layer techniques developed for III-nitride epistructures on sapphire substrates include those based on photoelectrochemical (PEC) wet etching, which exploits the selective photo-generation of free carriers in low-bandgap layers within a multilayer structure. A sophisticated application by Sharma et al involved fabrication of air-spaced Bragg mirrors, in which three sacrifical layers containing InGaN-based superlattices were removed [6]. This PEC method requires complicated superlatticebased epistructures for optimum application, and also requires intense illumination of samples at suitable wavelengths during the wet processing.

The background described has motivated a few previous studies on using the Ga-free nitride alloy AlInN as a sacrificial layer. General interest in this ternary has developed rapidly since 2003 for various applications in III-nitride device technology, including AlInN-GaN Bragg mirrors, field effect transistors, and barrier layers for $\mathrm{GaN}$ quantum wells [7]. AlInN shows lattice matching to hexagonal GaN on the basal plane at a composition of approximately $\mathrm{Al}_{0.83} \mathrm{In}_{0.17} \mathrm{~N}$ [8], and this particular composition is therefore of interest when targeting strain-free GaN-AlInN multilayers. AlInN also possesses the useful characteristic that it may be converted into a coherent oxide layer by electrochemical treatment (anodic oxidation), preferably conducted on intentionally doped n-type AlInN [7,9,10]. In the fabrication of optically pumped microdisk lasers, Simeonov and co-workers used such an oxidation process before 
removing the resulting oxide in an acid etch [10]. However, selective lateral etching of AlInN-GaN multilayers is also possible without preliminary oxidation. Wet etch routes to rudimentary 3-dimensional microstructures, using alkaline media for the removal of AlInN without photo-enhancement, were reported by Sillero et al [11] as well as our own group [12]. Subsequently we have switched our focus to the use of hot aqueous nitric acid for AlInN removal. Results reported earlier demonstrate the minimal effect of this etchant on the GaN crystal facets exposed during processing, and the fabrication of demonstrator microbridges in $\mathrm{GaN}$ with no intentional doping [13]. The work described here extends the microfabrication approach to more sophisticated epistructures containing conductive n-type GaN layers, achieved by means of silicon doping during growth. Electrical measurements made on the resulting doubly-supported microbridges show the potential for either electrostatic or Lorentz-force actuation of motion [1] in attainable geometries. This has particular significance as a means of driving cantilever and similar structures through a resonant condition in MEMS sensor applications.

\section{Experimental details}

Epitaxial multilayers were grown on 2-inch sapphire (0001) substrates by metal organic chemical vapour deposition (MOCVD) using an Aixtron 200-series singlewafer reactor. Procedures for doping of Si:GaN layers using di(tertiarybutyl)silane [DTBSi] and for GaN growth initiation, have been described in detail elsewhere $[14,15]$. The trimethylgallium molar flow rate was 66 micromoles $/ \mathrm{min}$, and the DTBSi molar flow rate was 4.7 nanomoles/min for thicker layers. AlInN was grown without intentional doping, under conditions described in earlier papers [16,17]. All GaN-AlInN-GaN trilayers were grown with the target of achieving lattice matching between the $\mathrm{GaN}$ and $\mathrm{AlInN}$ layers through control of the AlInN growth temperature, and hence composition. The setpoint temperature used was $770^{\circ} \mathrm{C}$ in the case of the structure with the 100-nm AlInN layer discussed in detail.

ICP etching was performed on a STS Ltd. Multiplex Pro system, using chlorine chemistry. The critical recipe parameters were $\mathrm{Cl}_{2}$ and $\mathrm{Ar}$ flow rates of 30 and 10 standard cubic centimetres/min respectively, a chamber pressure of 5 milliTorr, 600 $\mathrm{W}$ coil power, $200 \mathrm{~W}$ platen power, and a platen temperature of $30^{\circ} \mathrm{C}$. During the ICP etching, samples were masked either with patterns defined in single layers of photoresist (Rohm and Hass S1818), or with composite masks comprising S1805 photoresist deposited onto 600-nm layers of dense $\mathrm{SiO}_{2}$. In the latter case, the $\mathrm{SiO}_{2}$ was patterned in an additional reactive ion etch step before the ICP process. In situ monitoring of ICP etch rates used an Intellevation LEP300 reflectometer. Lateral wet etching to remove the AlInN was conducted as described previously [13], using a reflux apparatus to allow unattended operation over periods of $>20$ hours. The etch solution was aqueous nitric acid of 2 molar concentration. After the wet etch was complete, samples were given several rinses each with de-ionised water and then with isopropanol before final drying. Fabrication of electrodes used a lift-off process. 50$\mathrm{nm}$ layers of titanium, followed by $150-\mathrm{nm}$ layers of gold, were deposited in a CVC 601 sputter tool. The sputtering process was sufficiently energetic for these Ti-Au electrodes to show excellent ohmic behaviour without post-deposition annealing, and their properties are similar to those obtained by annealing conventional evaporated Tibased contacts [18]. 
Characterisation on as-grown epistructures was performed on wafer pieces from close to the central position, where growth rates and cumulative layer thicknesses were monitored by in situ reflectometry. Depth profiling to determine silicon atom concentrations (henceforth denoted by the abbreviation [Si]) by secondary ion mass spectrometry (SIMS) was performed at Cascade Scientific Ltd, U.K. Measured [Si] values were calibrated with reference to an ion-implanted Si:GaN standard, and have an estimated precision of $\pm 10 \%$. Analysis of the as-grown structure by Rutherford backscattering spectrometry (RBS) used procedures described previously [17]. Current-voltage (I-V) characteristics of fabricated microstructures were recorded using a Hewlett Packard HP4155A semiconductor parameter analyser, which swept the applied voltage over a pre-set range. Micromanipulator probes were placed on the contact pads. SEM imaging was performed on uncoated samples in an FEI Sirion instrument, at a standard beam accelerating voltage of $5 \mathrm{kV}$.

\section{Results and Discussion}

\subsection{Design and characterisation of epistructures}

The microbridges subject to electrical characterisation were fabricated from a GaNAlInN-GaN trilayer with an AlInN layer of $100 \mathrm{~nm}$ nominal thickness, the full layer sequence of which is illustrated in Fig. 1. In situ reflectometry during the MOCVD process gave feedback on the cumulative thickness of the AlInN layer, as well as more precise growth rate data on the thicker GaN layers [15]. However, RBS provided a more accurate value for the AlInN thickness, and gave a best-fit thickness of $92 \mathrm{~nm}$. The InN fraction measured by RBS was $16.7 \%$, which is within experimental error limits of the value of $17.1 \%$ required for lattice matching with $\mathrm{GaN}$ [8]. Fig. 1 shows that the structure was designed with an $\sim 55 \mathrm{~nm}$ surface contact layer, in which the nominal [Si] value was twice that in the $\mathrm{Si}: \mathrm{GaN}$ immediately below. SIMS measurements indicated a [Si] value of $5.7 \times 10^{18} \mathrm{~cm}^{-3}$ in this thicker Si:GaN layer. The GaN below the AlInN layer was also doped, in order to facilitate electrostatic actuation of suspended beam structures as discussed further in section 3.3. The epistructure as described formed one of a set with AlInN layer thicknesses of 50, 150 and $300 \mathrm{~nm}$.

\subsection{Microstructure fabrication}

The ICP process produced steeply sloping sidewalls, and showed an approximate 1:1 etch rate selectivity between $\mathrm{GaN}$ and hardened photoresist. Relatively shallow etching of microstructures to the GaN layer below the AlInN layer used photoresist masks of thickness $1.8 \mu \mathrm{m}$. For deeper etching to the sapphire we used $\mathrm{SiO}_{2}$-photoresist composite masks. Reflectance monitoring indicated ICP etch rates of 580 and 400 $\mathrm{nm} / \mathrm{min}$ for GaN and AlInN respectively. The etch rate of the AlInN was determined using the GaN-AlInN-GaN structure with a 300-nm ternary layer. Other dry etch conditions can give much higher selectivites between GaN and lattice-matched AlInN, which may be useful in different applications exploiting AlInN as an etch stop or marker layer. For example, Sillero and co-workers reported etch rates for AlInN lower than those of $\mathrm{GaN}$ by factors of up to 17 [reference 11].

The standard microstructure defined by ICP etching was a bridge of $20 \mu \mathrm{m}$ length and $4 \mu \mathrm{m}$ width, supported by $50 \mu \mathrm{m} \times 50 \mu \mathrm{m}$ square anchor posts, as illustrated in Fig. 2 (a). Some microstructures were made by etching down to the sapphire substrate around 
the microbridge array, giving complete electrical isolation of individual bridges, while the etch was stopped in the lower GaN layer in other cases. Following the ICP step and removal of residual photoresist, the samples were wet etched in hot aqueous nitric acid. We reported in reference 13 the effect of this lateral etching process on cylindrical micropillars fabricated from undoped GaN-AlInN-GaN trilayers. Structures with AlInN layers of high crystallographic quality showed anisotropic etching, such that form of the ternary layer tended towards a hexagonal prism. Average lateral etch rates, measured on a few different epistructures and referenced to the midpoints of the hexagon facets, were in the range 140-210 nm/hr. The structure with Si:GaN layers used for microbridge fabrication showed similar anisotropy, and an AlInN etch rate at the lower end of the previously observed range. There is no evidence from optical microscope inspection for conversion of AlInN to a persistent oxide layer, as can be intentionally formed by electrochemical oxidation of $\mathrm{AlInN}[7,9,10]$. Reference 9 illustrates that this anodic oxidation process produces a demarcation between the oxidised and as-grown AlInN easily visible by optical microscopy. We conclude that, although the nitric acid etchant was selected in part because of its oxidising properties, any oxide formed dissolves quickly under our processing conditions.

Figs. 2 (b) and 2 (c) show SEM images of two microbridges subjected to 28 hrs of wet etching. The ICP etch step had been continued down to the sapphire substrate in the case of Fig. 2 (c). Lateral wet etching of the AlInN layer occurred at the edges of the square anchor posts as well as under the microbridges, as evidenced in Fig. 2 (b). However, an area of intact AlInN $1800 \mu \mathrm{m}^{2}$ or more in extent remained at both ends of the microbridges studied. Confirmation of complete removal of the AlInN layer under the narrow bridges was provided by the bowing observed in the SEM images. Upwards and downwards deflections, the latter of which is clearly shown in Fig. 2 (c), occurred with similar frequency, confirming significant net compressive strain in the Si:GaN layers. This effect was previously observed in analogous undoped structures, and the minimum strain required for deformation was related to Euler's critical buckling criterion [13]. The origin of a strain of sufficient magnitude is not entirely clear given that the RBS compositional measurement on the new structure suggests minimal lattice mismatch between the AlInN layer and GaN. However, the thermal expansion mismatch between sapphire and III-nitrides is expected to contribute $\sim 0.2 \%$ compressive strain to structures grown heteroepitaxially on this substrate [19], and ongoing work is examining structures with AlInN sacrificial layers grown on freestanding GaN substrates.

\subsection{Electrical measurements on fabricated microstructures and prospects for MEMS actuation}

Deposition of large-area metal contact pads onto the anchor posts of microbridges allowed I-V characteristics to be measured by probing. Intact microbridges all showed linear I-V characteristics, which were very similar within each wafer area. Fig. 3 (a) shows a typical characteristic from a microstructure etched down to the sapphire substrate, which corresponds to an ohmic resistance of $1050 \Omega$. It is informative to compare this measured resistance with that calculated for an individual microbridge, based on the idealised geometry, and reasonable assumptions on the carrier concentration and mobility in the Si:GaN. The calculation now presented assumes there is no significant parallel conduction path through the anchor posts, as verified for low applied voltages by measurements to be discussed. The contact resistance of the 
Ti-Au pads, and series resistance contribution of the Si:GaN layers in the anchor posts, are also assumed to be negligible in the calculation.

The free carrier concentration in the Si:GaN cannot be assumed to equal the [Si] value measured by SIMS, because of the finite compensation ratios common to all intentionally doped n-type GaN [20]. SIMS measurements on a reference Si:GaN sample with no AlInN interlayer indicated a [Si] value of $4.1 \times 10^{18} \mathrm{~cm}^{-3}$, whereas Hall measurements on the same sample gave a free carrier concentration of $2.6 \times 10^{18} \mathrm{~cm}^{-3}$ at room temperature [14]. Assuming that the same scaling factor applies to the trilayer sample, we estimate a carrier concentration in the microbridge of $3.6 \times 10^{18} \mathrm{~cm}^{-3}$ below the thin contact layer. At such high dopant levels room-temperature mobilities in $\mathrm{GaN}$ are governed by ionised impurity scattering, and a relation between mobility and carrier concentration in 2- $\mu \mathrm{m}$ Si:GaN layers from reference 14 gives a mobility estimate of $230 \mathrm{~cm}^{2} \mathrm{~V}^{-1} \mathrm{~s}^{-1}$ at $3.6 \times 10^{18} \mathrm{~cm}^{-3}$. The assumptions outlined so far lead to a Si:GaN resistivity of $7.5 \mathrm{~m} \Omega \cdot \mathrm{cm}$. To approximate the contribution of the thin highly doped contact layer we model the microbridge as uniformly doped Si:GaN, but with a thickness $10 \%$ greater than the sum of thicknesses for the two layers above the AlInN in Fig. 1, and numerically equal to $620 \mathrm{~nm}$. Thus a resistance of $610 \Omega$ is predicted for a bar of idealised dimensions (20 $\mu \mathrm{m}$ length, $4 \mu \mathrm{m}$ width, and the effective thickness just stated). The measured resistances of the microbridges discussed were some $70 \%$ higher, but a resistance value larger than the calculated value is evidence against any important parallel conduction path. In practice several factors could explain experimental resistance values higher than the calculated value. For example, under the MOCVD growth conditions used the GaN growth rate decreases by approximately $20 \%$ going from the centre to the edge of the rotating wafer. Therefore individual microbridges may have cross-sectional areas significantly lower than assumed in the calculation.

The small magnitude of the current passed through the parallel conduction path through the anchor posts at low voltages was verified by I-V measurements on microstructures where the bridge fractured during processing (principally during the lift-off patterning of the electrodes). Fig 3 (b) shows a typical I-V characteristic from such a pair of disconnected anchor posts, and the inset shows the conduction path possible through the AlInN layers. In view of the small currents measured, contributions from surface leakage cannot be ruled out. Arguments against gross conversion of the AlInN to an insulating oxide phase were presented earlier. The current passed at $+2 \mathrm{~V}$ is below $8 \mu \mathrm{A}$, ie. a factor of $>200$ lower than the analogous current in Fig. 3 (a). Between +5 and $+6 \mathrm{~V}$ the differential resistance is $\sim 7.2 \mathrm{k} \Omega$. Little information is available on the vertical conductivity of AlInN layers grown without deliberate doping, which may be assumed to have an n-type background. However, it has been found very challenging to obtain high conductivities in any AlN-rich alloys, owing to the formation of compensating point defects and traps [21, 22]. Therefore the absence of significant vertical conduction through these AlInN layers is understandable. The conduction band offsets at the heterointerfaces will contribute to the non-linearity of the I-V characteristic shown in Fig. 3 (b). The flat-band offset between $\mathrm{GaN}$ and AlInN can be estimated as $\sim 0.7 \mathrm{eV}$, assuming an AlInN bandgap at the lattice-matched composition of $4.4 \mathrm{eV} \mathrm{[16],} \mathrm{and} \mathrm{a} \mathrm{conduction-to-valence-band}$ offset ratio of 2.8:1 [23]. A fuller analysis would need to consider polarisation fields, but it may be noted that these run in opposing directions, referenced to the current flow, in the two AlInN layers. 
In order to assess the robustness of intact microbridges, a few examples were tested at higher applied voltage ranges than shown in Fig. 3 (a). In a typical case, the bridge showed nearly linear I-V characteristics up to $\pm 4 \mathrm{~V}$, but a significant reduction of gradient occurred at the extremes of a $\pm 6 \mathrm{~V}$ scan. This reversible behaviour can be attributed to a reduction in carrier mobility under self-heating. Subsequent scans at \pm 8 and $\pm 10 \mathrm{~V}$ resulted in the I-V characteristic changing irreversibly, and the behaviour switching to resemble that of bridges broken during the microfabrication steps. SEM gave direct confirmation that bridges fractured at these higher applied voltages. More work is necessary to understand the failure mechanism, which will be complicated by the pre-existing compressive strain in the current structures. However, it may be noted that $\sim 10 \mathrm{~mW}$ was typically generated by Joule heating in microbridges at the onset of a permanent change to their I-V characteristics.

Concerning possibly actuation mechanisms for microstructures with geometries similar to described, two methods standard in MEMS technology, electrostatic and Lorentz force actuation [1], should both prove effective. The calculated Lorentz force on a microbridge $20 \mu \mathrm{m}$ in length and passing a current of $2 \mathrm{~mA}$ in a crossed magnetic field of $1 \mathrm{~T}$ is $40 \mathrm{pN}$. In the case of electrostatic actuation, the lower Si:GaN layer can serve as a counterelectode at fixed potential, and the fabrication technique means that this is separated from a moving element by a distance similar to the thickness of the AlInN sacrificial layer, if bowing effects are eliminated. Electrostatic actuation also requires minimal conduction through the unetched AlInN in anchor posts, as we observed experimentally. The calculated force on a bridge $20 \mu \mathrm{m}$ by 4 $\mu \mathrm{m}$ separated by a constant $0.1 \mu \mathrm{m}$ from a ground plane, and with an applied potential difference of $2 \mathrm{~V}$, is $142 \mathrm{pN}$, approximately 3.5 times the Lorentz force just quoted.

\section{Summary and conclusions}

Microscale air bridges have been fabricated in conductive Si:GaN, grown above a sacrificial AlInN layer. An MOCVD technique was used to grow GaN-AlInN-GaN epistructures on sapphire substrates, containing AlInN layers of various thicknesses up to $300 \mathrm{~nm}$, and close to the composition needed for lattice matching with GaN. The plan-view form of the microbridges was defined by photolithography and vertical ICP etching using chlorine chemistry. Bridges discussed in detail have a length of $20 \mu \mathrm{m}$, a width of $4 \mu \mathrm{m}$, a thickness of $\sim 560 \mathrm{~nm}$, and were fabricated from an epistructure containing an AlInN layer of $100 \mathrm{~nm}$ nominal thickness. During fabrication the AlInN etched at $\sim 70 \%$ of the rate of GaN under the ICP conditions. Wet etching in hot nitric acid was then used to selectively remove the AlInN, and leave structures with an air gap below a Si:GaN beam anchored between two support posts. SEM showed deflections of the bridges consistent with net compressive strain, to which thermal expansion mismatch with the sapphire substrate contributes. Deposition of electrodes on the anchor posts allowed the I-V characteristics of the microbridges to be measured. Occasional breakages proved beneficial in allowing direct investigation of a parallel conduction path through AlInN layers, which was shown to be of minor importance at low applied voltages. The microbridges themselves showed linear I-V characteristics up to current densities of $\sim 2 \times 10^{5} \mathrm{~A} \mathrm{~cm}^{-2}$, but underwent irreversible changes at higher currents, eventually fracturing. The ohmic resistances of the microbridges were in the $1 \mathrm{k} \Omega$ range. These showed agreement within a factor of two with predicted values based on the idealised geometry, SIMS measurements of the silicon atom 
concentration, plus reasonable assumptions on the carrier mobility and compensation ratio. Either electrostatic or Lorentz force actuation should be possible in microstructures with geometries and electrical characteristics similar to those reported.

\section{Acknowledgement}

The authors thank Dr K. Lorenz, Instituto Tecnológico e Nuclear, Sacavém, Portugal, for the RBS measurement. Funding from the UK EPSRC supported C. Xiong's work at the Institute of Photonics as an academic visitor.

\section{References}

[1] R. Cimalla, J. Pezoldt, O. Ambacher: J. Phys. D: Appl. Phys. 40, 6386 (2007)

[2] K.J. Hjort: Micromech. Microeng. 6, 370 (1996)

[3] Z. Yang, R.N. Wang, S. Jia, D. Wang, B.S. Zhang, K.M. Lau, J. Chen: Appl. Phys. Lett. 88, 041913 (2006)

[4] T. Zimmermann, M. Neuburger, P. Benkart, F.J. Hernández-Guillén, C. Pietzka, M. Kunze, I. Daumiller, A. Dadgar, A. Krost, E. Kohn : IEEE Electron. Dev. Lett. 27, 309 (2006)

[5] H.W. Choi, K.N. Hui, P.T. Lai, P. Chen, X.H. Zhang, S. Tripathy, J.H. Ting, S.J. Chua: Appl. Phys. Lett. 89, 211101 (2006)

[6] R. Sharma, E.D. Haberer, C. Meier, E.L. Hu, S. Nakamura: Appl. Phys. Lett. 87, 051107 (2005)

[7] R. Butté, J.-F. Carlin, E. Feltin, M. Gonschorek, S. Nicolay, G. Christmann, D. Simeonov, A. Castiglia, J. Dorsaz, H.J. Buehlmann, S. Christopoulos, G. Baldassarri Höger von Högersthal, A.J.D. Grundy, M. Mosca, C. Pinquier, M.A. Py, F. Dermangeot, J. Frandon, P.G. Lagoudakis, J.J. Baumberg, N. Grandjean: J. Phys. D: Appl. Phys. 40, 6328 (2007)

[8] K. Lorenz, N. Franco, E. Alves, I.M. Watson, R.W. Martin, K.P. O’Donnell: Phys. Rev. Lett. 97, 085501 (2006)

[9] J. Dorsaz, H.-J. Bühlmann, J.-F. Carlin, N. Grandjean, M. Ilegems: Appl. Phys. Lett. 87, 072102 (2005)

[10] D. Simeonov, E. Feltin, A. Altoukhov, A. Castiglia, J.-F. Carlin, R. Butté, N. Grandjean: Appl. Phys. Lett. 92171102 (2008)

[11] E. Sillero, D. Lopéz-Romero, F. Calle, M. Eickhoff, J.F. Carlin, N. Grandjean, M. Ilegems: Microelectron. Eng. 84, 1152 (2007)

[12] F. Rizzi, K. Bejtka, P.R. Edwards, R. W. Martin, I.M. Watson: J. Cryst. Growth, 300, 254 (2007)

[13] I.M. Watson, C. Xiong, E. Gu, M.D. Dawson, F. Rizzi, K. Bejtka, P.R. Edwards, R.W. Martin: Proc. SPIE, 6993, 69930E (2008)

[14] C.J. Deatcher, C. Liu, M.-G. Cheong, L.M. Smith, S. Rushworth, A. Widdowson, I. M. Watson: Chem. Vap. Dep. 10, 187 (2004)

[15] C. Liu, I.M. Watson: Semicond. Sci. Technol. 22, 629 (2007)

[16] K. Wang, R.W. Martin, D. Amabile, P.R. Edwards, S. Hernandez, E. Nogales, K.P. O’Donnell, K. Lorenz, E. Alves, V. Matis, A. Vantomme, D. Wolverson, I.M. Watson: J. Appl. Phys. 103, 073510 (2008)

[17] K. Lorenz, N. Franco, E. Alves, S. Pereira, I.M. Watson, R.W. Martin, K.P. O'Donnell: J. Cryst. Growth 310, 4058 (2008)

[18] B. Boudart, S. Trassaert, X. Wallart, J.C. Pesant, O. Yaradou, D. Théron, Y. Crosnier, H. Lahreche, F. Omnes: J. Electron. Mater., 29, 603 (2000)

[19] M. Seyboth, S.-S. Schad, M. Scherer, F. Habel, C. Eichler, M. Kamp, V. Scwegler: J. Mater. Sci.: Mater. Electronics, 13, 659 (2002). 
[20] C. Mavroidis, J.J. Harris, R.B. Jackman, I. Harrison, B.J. Ansell, Z. Bougrioua, I. Moermann: J. Appl. Phys. 91, 9835 (2002)

[21] S. Keller, P. Cantu, C. Moe, Y. Wu, S. Keller, U.K. Mishra, J.S. Speck, S.P. DenBaars: Japan. J. Appl. Phys. 44, 7227 (2005)

[22] T. Ive, O. Brandt, H. Kostial, T. Hesjedal, M. Ramsteiner, K.H. Ploog: Appl. Phys. Lett. 85, 1970 (2004)

[23] G. Franssen, T. Suski, M. Krysko, A. Khachapuridze, R. Kudrawiec, J. Misiewicz, A. Kaminska, E. Feltin, N. Grandjean: Appl. Phys. Lett. 92, 201910 (2008) 


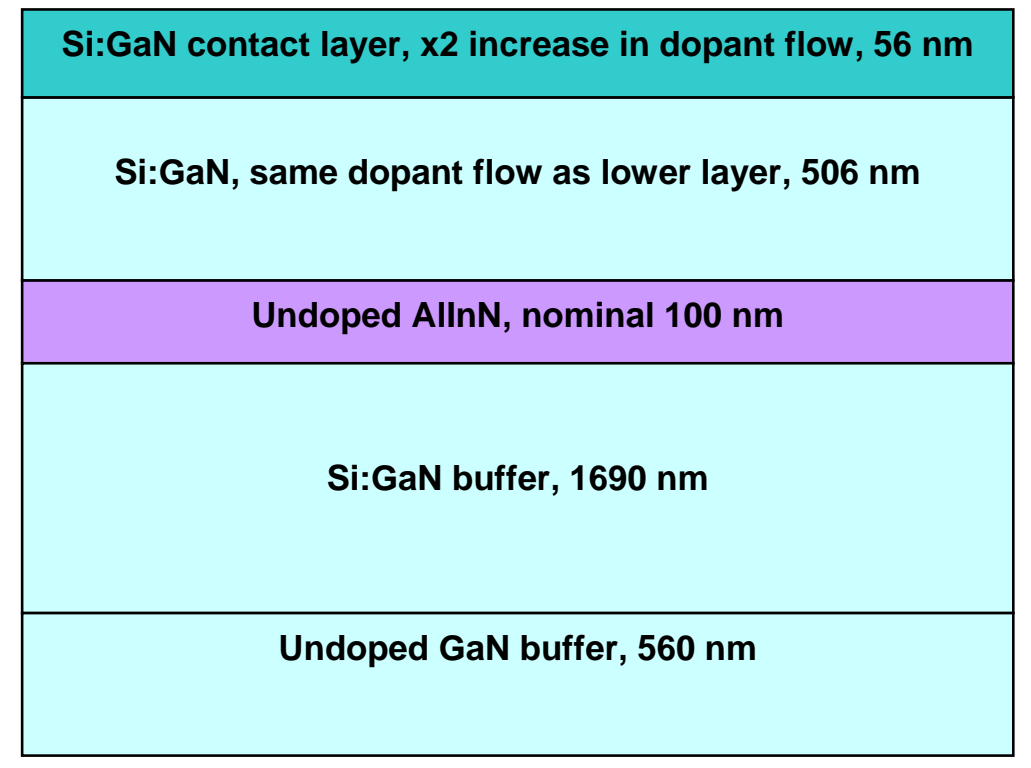

Fig. 1 - Schematic showing the layer sequence and thicknesses in the epistructure used to fabricate microbridges. The GaN layer thicknesses shown correspond to the growth rate of $2.25 \mu \mathrm{m} / \mathrm{hr}$ measured by in situ reflectometry for the wafer centre. The sapphire substrate, and the low-temperature $\mathrm{GaN}$ nucleation layer of $\sim 25 \mathrm{~nm}$ thickness, are omitted. 


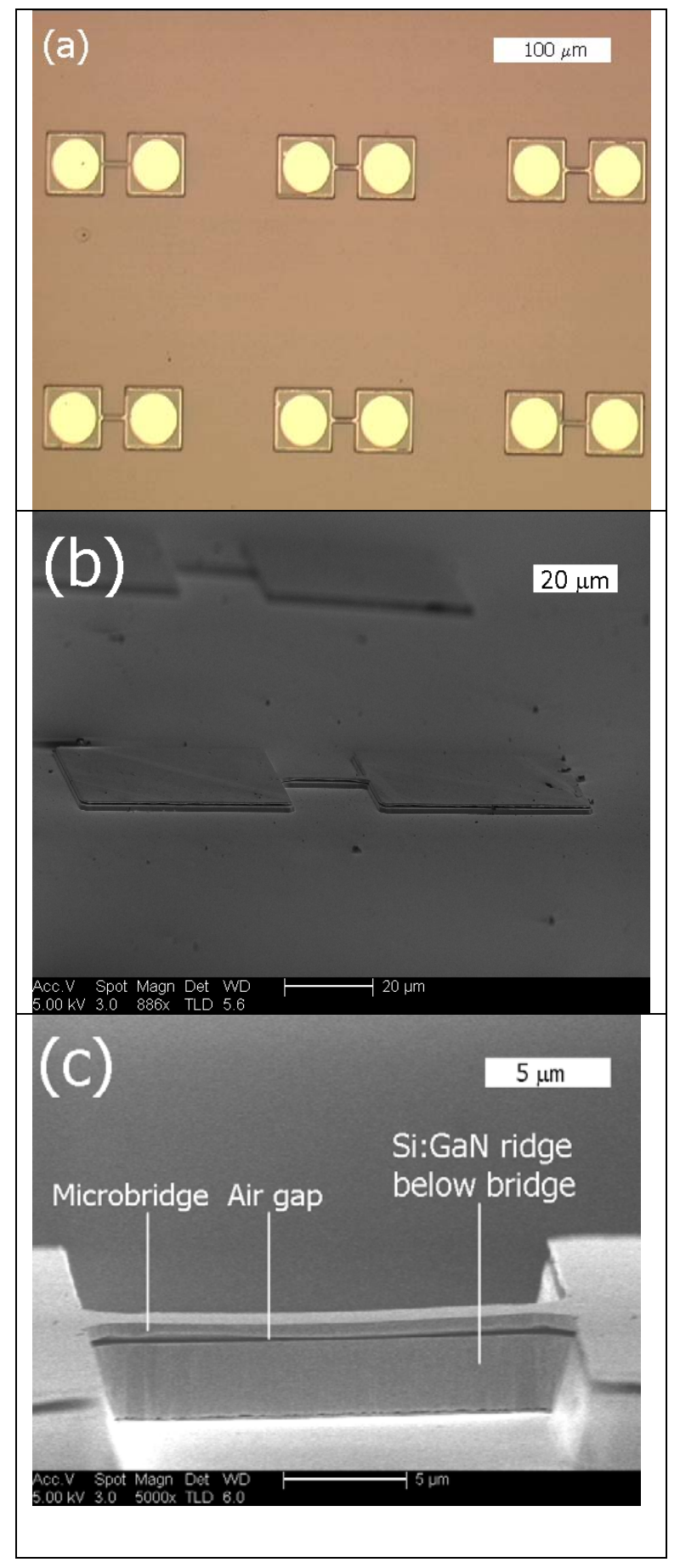

Fig. 2 - Images of fabricated microbridges, with air gaps introduced by removing the AlInN layer. Fig 2 (a) is an optical micrograph of an array of bridges after deposition of metal pads on the $50-\mu \mathrm{m}$ square anchor posts. Bridges at the top left and bottom right of this field are broken. Figs. 2 (b) and (c) show SEM images of intact microbridges before the metal pad deposition, at tilts of $82^{\circ}$ and $86^{\circ}$ respectively. In the case of Fig. 2 (b) ICP etching was stopped in the GaN below the AlInN. In Fig. 2 (c), the bridge shows downwards deflection, and ICP etching was continued down to the sapphire around the bridge and support posts. 


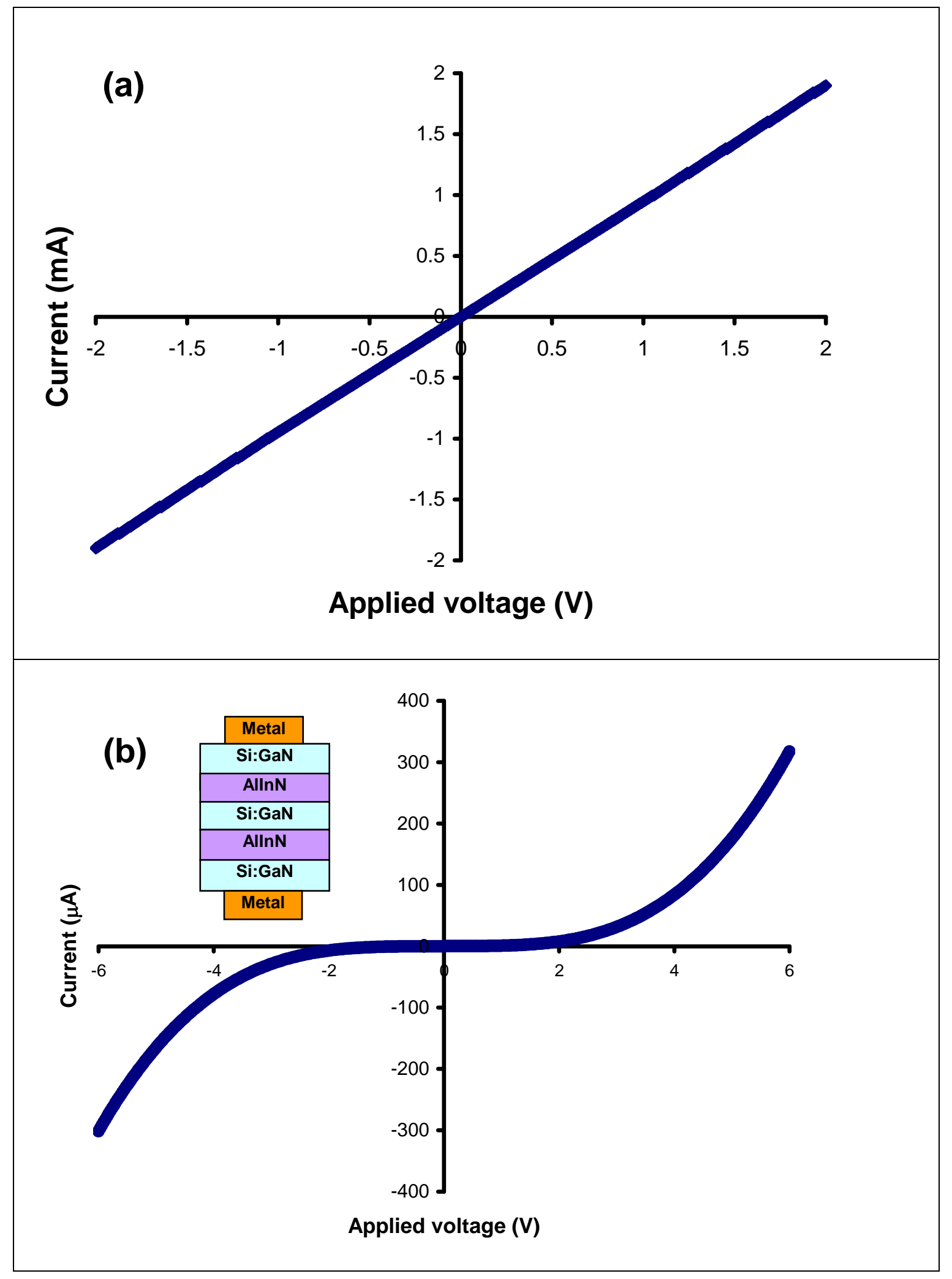

Fig. 3 - Typical I-V characteristics for an intact microbridge at low applied voltage (a) and a microstructure with a broken bridge (b). The inset in Fig. 3 (b) shows the current path through GaN-AlInN heterojunctions, which is present as a minor conduction path in parallel with intact bridges. Both structures were etched down to the sapphire substrate. 